\title{
19. THE SOFT X-RAY BACKGROUND
}

\author{
H. FRIEDMAN, G. FRITZ, and S. D. SHULMAN \\ E. O. Hulburt Center for Space Research, Naval Research Laboratory, \\ Washington, D.C. 20390, U.S.A. \\ and \\ R. C. HENRY \\ E. O. Hulburt Center for Space Research, and The Johns Hopkins University, \\ Baltimore, Md. 21218, U.S.A.
}

\begin{abstract}
A survey of soft X-ray background observations in the $0.1-10 \mathrm{keV}$ range is presented. In the region above $1 \mathrm{keV}$, recent results on point $\mathrm{X}$-ray sources are discussed and their integrated contribution to the diffuse background is estimated. However, the average luminosity of various classes of extragalactic X-ray sources is still not sufficiently well known to permit this estimate to be made with any certainty. A discussion is given of recent observations at energies below $1 \mathrm{keV}$ where the effects of interstellar absorption are important. It is argued that although some fraction of the background radiation in the $0.1-1 \mathrm{keV}$ range must be galactic in origin, there is still substantial evidence for an extragalactic component. Proposed theories for generating both the galactic and extragalactic X-ray background are briefly reviewed.
\end{abstract}

\section{Introduction}

Over the past decade the $\mathrm{X}$-ray background radiation has been studied almost as intensively as have discrete sources. From $1 \mathrm{keV}$ to $1 \mathrm{MeV}$, the spectrum is fairly well defined, and general isotropy in the $1-20 \mathrm{keV}$ region has been established to a considerable degree. Above $20 \mathrm{keV}$ the spectrum approximates a power law with an index of about -2.3 for the differential photon flux. At lower energies the spectrum flattens, and the index, from 2 to $8 \mathrm{keV}$, falls to about -1.4 . For the lowest observed energies $(100 \mathrm{eV}$ to $1 \mathrm{keV})$, the measurement techniques introduce large uncertainties. Nevertheless, the body of data accumulated in the past three or four years indicates a significant excess over any simple extrapolation of the higher energy spectrum. In this review we confine our attention to the range 0.1 to $10 \mathrm{keV}$. The work has been carried out with rocket- and satellite-borne proportional counters. As an Appendix, we include a discussion of the instrumental problems specific to soft X-ray measurements with proportional counters.

The background may be composed of the integrated contributions of discrete sources and of truly diffuse X-rays generated in interstellar or intergalactic space. From the wealth of data on discrete sources that is now available in the soft X-ray range, we shall assess the contribution of discrete sources. At the very lowest energies $(100 \mathrm{eV}$ to $1 \mathrm{keV}$ ), it is still debatable whether the observed background is partly extragalactic or entirely local in origin. Our most recent results favor an extragalactic component which can be distinguished from soft $X$-rays generated within the galaxy. Intrinsic to any understanding of the $\mathrm{X}$-ray background is the possible role of intergalactic gas; we shall discuss X-ray observations of clusters of galaxies which offer important clues in this regard. 


\subsection{THE X-RAY LUMINOSITY OF NORMAL GALAXIES}

A few years back, estimates of the luminosity of our galaxy (Friedman et al., 1967), based on a sampling of some 30 sources, led to a value of about $7 \times 10^{39} \mathrm{erg} \mathrm{s}^{-1}$. In arriving at this figure, it was assumed that the observed sources are grouped in the nearest spiral arms and represent only about $2 \%$ of the volume of the galactic disk. Subsequent observations of spectral extinction below $1 \mathrm{keV}$ implied that the observed sources were distributed over a larger range of distances and represented a larger sample of the volume of the galaxy. Although fewer in number, the deduced average luminosity of the sources was higher. The new estimate of $L_{\mathrm{gal}}$ became $2-3 \times 10^{39}$ $\mathrm{erg} \mathrm{s}^{-1}$. Andromeda is a giant spiral similar to the Milky Way. The flux (2 to $\left.7 \mathrm{keV}\right)$ observed by UHURU leads to a luminosity of about $2 \times 10^{39} \mathrm{erg} \mathrm{s}^{-1}$, in good agreement with earlier estimates for our galaxy. From an observation of the Large Magellanic Cloud by Mark et al. (1969), a luminosity of about $4 \times 10^{38} \mathrm{erg} \mathrm{s}^{-1}$ was deduced, consistent with the roughly tenfold lower stellar content than that of the Milky Way.

The UHURU catalog now lists 125 sources, about two-thirds of which lie close to the galactic plane and the remaining one-third at latitudes $> \pm 20^{\circ}$. Amongst the galactic sources we can identify supernova remnants such as the Crab Nebula, the Supernova of 1572 , and Cas $\mathrm{A}$. For all of these relatively younger supernovae, the X-ray luminosities are in the range $10^{36}-10^{37} \mathrm{erg} \mathrm{s}^{-1}(1$ to $10 \mathrm{keV})$. A second, perhaps larger, class of sources are binary systems in which a compact object - white dwarf, neutron star, or, perhaps, a black hole - accretes gas from a large stellar companion. Cyg X-1, Cen X-3, and $2 \mathrm{U} 1702+35$ in Hercules are representative of this group. From a combination of $21-\mathrm{cm}$ emission and X-ray absorption studies, the estimated distance to Cyg X-1 is about 1000 parsec and its luminosity is, therefore, also about $10^{36} \mathrm{erg} \mathrm{s}^{-1}$. It appears that this luminosity may represent close to the average value for most of the roughly 100 sources detected thus far in the galaxy. Their sum would amount to about $10^{38} \mathrm{erg} \mathrm{s}^{-1}$, about an order of magnitude less than our previous estimates of the galactic luminosity. However, there is evidence of discrete sources in the Magellanic Clouds with luminosities of $10^{38} \mathrm{erg} \mathrm{s}^{-1}$ and one example of a luminosity as great as $10^{39} \mathrm{erg} \mathrm{s}^{-1}$. In the region of the galactic bulge of our galaxy there appear to be several sources as powerful as $10^{38} \mathrm{erg} \mathrm{s}^{-1}$. We may speculate that the bulk of the galactic luminosity could be made up of just a few sources with luminosities in the range of $10^{38} \mathrm{erg} \mathrm{s}^{-1}$, perhaps a few hundred sources in the $10^{36}-10^{38} \mathrm{erg} \mathrm{s}^{-1}$ range and, possibly, thousands of sources with average luminosities between $10^{35}$ and $10^{36} \mathrm{erg} \mathrm{s}^{-1}$.

In any case, the direct observation of Andromeda supports the estimate that the $\mathrm{X}$-ray flux from normal galaxies is about $10^{39} \mathrm{erg} \mathrm{s}^{-1}$. The contribution of all such galaxies, assuming a space density of $0.03 \mathrm{Mpc}^{-3}$, amounts to between $10^{-1}$ and $10^{-2}$ of the observed background of $2 \times 10^{-7} \mathrm{erg} \mathrm{cm}^{2} \mathrm{~s}$ sterad. It is possible that evolutionary effects could provide a much higher X-ray luminosity in earlier epochs than at present, but we are a long way from the observational capability to test such a hypothesis. 


\subsection{Pulsars}

The pulsar NP 0532 in the Crab Nebula radiates about $10^{36} \mathrm{erg} \mathrm{s}^{-1}$ from 1 to $10 \mathrm{keV}$. Several attempts have been made to estimate the contribtuion of supernovae to the diffuse background on the assumption that the Crab is a typical supernova remnant and that the average rate of appearance of supernovae is about one per $100 \mathrm{yr}$ per normal galaxy. If a new pulsar is endowed with $10^{52} \mathrm{erg}$ of rotational kinetic energy and if the conversion efficiency to $\mathrm{X}$-ray emission is about one percent, $10^{50} \mathrm{erg}$ would be available in the pulsar lifetime. Averaged over time, the contribution from pulsars alone would yield a galactic luminosity of $10^{41} \mathrm{erg} \mathrm{s}^{-1}$ and the combined X-ray background emission from all galaxies would be equal to the observed diffuse background.

However, the estimate of the initial energy of rotation is very uncertain. Some authors prefer as high a value as $1.5 \times 10^{53} \mathrm{erg}$ (Gunn and Ostriker, 1969) and others as little as $5 \times 10^{49}$ (Woltjer, 1969). To avoid exceeding the observed upper limit on anisotropy, an average emission lifetime of 4 days per pulsar would be required. Thus far, only upper limit flux results are available from X-ray observations directed toward known supernova events in distant galaxies. Bradt et al. (1968) set an upper limit of $2 \times 10^{42} \mathrm{erg} \mathrm{s}^{-1}$ for a supernova in NGC 4254. Since the observation was made 40 days after the phase of maximum light, they could only deduce that the emission lifetime would need to be shorter than $0.1 \mathrm{yr}$ for the event to have decayed before observation, or greater than $5 \mathrm{yr}$ for the average emission to be less than the detector sensitivity. For four supernova events observed from the OSO III satellite in 1967 and 1968, Ulmer et al. (1972) found only upper limits on the fluxes which give upper limits on total energies of from $10^{50}$ to $10^{51} \mathrm{erg}$. The observations, therefore, do not yet answer the supernova question in any useful way.

\subsection{X-RAYS FROM RADIO GALAXIES, SEYFERT GALAXIES, QUASARS, AND CLUSTERS}

If normal galaxies cannot account for the $\mathrm{X}$-ray background, can a much smaller number of powerful radio galaxies, Seyferts, or quasars be responsible? Among the distant galaxies that have been observed as X-ray sources are M 87, Cen A, NGC 1275, NGC 4151, and the quasar 3C 273. The claims for identification are, of course, based on the uniqueness of these sources as radio galaxies and Seyferts rather than on any precise location of the X-ray source. At its apparent red shift distance of $630 \mathrm{Mpc}$, $3 \mathrm{C} 273$ radiates $\sim 10^{46} \mathrm{erg} \mathrm{s}^{-1}$ and is the most powerful $\mathrm{X}$-ray source detected thus far. Estimates of integrated background, based on the assumption that some of these galaxies are typical members of their class, can lead to embarrassingly high values of the background. This is true for quasars, if 3C 273 is typical, and for Seyferts, if NGC 1275 is representative. However, NGC 4151 is far weaker than NGC 1275, and NGC 1068, a strong infrared Seyfert galaxy which was predicted to be a powerful X-ray emitting object was undetected by UHURU and is probably one to two orders of magnitude weaker than NGC 1275. Clearly, our sampling to date does not permit any quantitative assessment of the integral contribution of quasars, Seyferts and strong 
radio galaxies such as $\mathbf{M} 87$. Perhaps we have detected thus far only the most unusual specimens of these classes of unusual galaxies.

Observations of clusters of galaxies with rockets and by the UHURU satellite now include several prominent clusters such as Virgo, Coma, and Perseus. It is not entirely clear how much of the observed flux is attributable to one or two relatively powerful radio galaxies in any particular cluster, for example, $M 87$ in Virgo, or to the larger number of galaxies that make up each cluster. In both Coma and Virgo (minus the contribution of $M$ 87) the luminosity per galaxy appears to be about 20 times that of our own galaxy. It is possible that the X-rays originate largely in hot intracluster gas rather than in the individual galaxies, but the observations are still too primitive to make any clear determination of the nature of the source.

\subsection{ISOTROPY}

Any model which attributes the diffuse background (1-10 keV) to discrete sources must be consistent with the limits on anisotropy. Schwartz et al. (1970) obtained X-ray background data from OSO III which covered about $50 \%$ of the sky and showed no rms intensity fluctuations larger than $3 \%$ on the scale of their beam width $\left(\sim 30^{\circ}\right)$. Wolfe and Burbidge (1970) compared these observations with theoretically expected fluctuations corresponding to clusters or super-clusters of galaxies. Their results argued against any hierarchical universe models in which the distribution of X-ray sources corresponded to the cosmological mass distribution.

Fabian and Sanford (1971) have observed nine regions of sky and conclude from the observed isotropy that there must be at least $10^{7}$ sources of equal apparent brightness over the entire sky. The corresponding number for sources of equal absolute intensity is $\sim 10^{9}$, which implies a number density exceeding that for Seyfert galaxies. Schwartz et al. (1971) believe that the Fabian and Sanford experimental limit should be lowered considerably and suggest a reduction to about $10^{4}$ sources over the entire sky. Furthermore, from an autocorrelation of their count rate as a function of angle, they find results consistent with random statistical scatter and inconsistent with a supercluster model and some cluster models.

\section{Recent Observations of the (0.1-1 keV) X-Ray Background}

In the 1-10 $\mathrm{keV}^{r}$ region there is very good agreement between various groups (Boldt et al., 1969; Ducros et al., 1970; Bunner et al., 1971; Shukla and Wilson, 1971; Shulman et al., 1971; and Toor et al., 1972) as to the spectrum of the diffuse background. A power law representation, $A E^{-\gamma}$ photons $\left(\mathrm{cm}^{2} \mathrm{~s} \mathrm{ster} \mathrm{keV}\right)^{-1}$, is fit by these groups to their data. $A$ is found to be in the range $8-11$, and $\gamma$ is between 1.3 and 1.4 .In the soft $X$-ray region, $10-100 \AA(0.1-1 \mathrm{keV})$ there is general agreement that the flux near $44 \AA$ exceeds that expected from an extrapolation of the spectrum in the $1-10 \mathrm{keV}$ region. The original interpretation attributed most of the excess at the galactic pole to an extragalactic orgin (Bowyer et al., 1968; Henry et al., 1968). However, Henry et al. (1968) also observed a significant flux of $44 \AA$ X-rays from 
the galactic plane. The soft $\mathrm{X}$-ray emission from the galactic plane is now well established (Henry et al., 1971; Bunner et al., 1971; Palmieri et al., 1971, Hayakawa et al., 1971 and Yentis et al., 1972) and this emission almost certainly requires a galactic origin since the mean free path for $44 \AA \mathrm{X}$-rays in the interstellar medium is on the order of only $100 \mathrm{pc}$. In a recent observation, the Wisconsin group (McCammon et al., 1971) found no evidence for absorption of the diffuse background by the Small Magellanic Cloud (SMC), and placed an upper limit of $25 \%$ on the fraction of $44 \AA$

TABLE I

Soft X-ray background intensities

\begin{tabular}{llllll}
\hline $\begin{array}{l}\text { Wavelength } \\
{[\AA]}\end{array}$ & $\begin{array}{l}\text { Energy } \\
{[\mathrm{keV}]}\end{array}$ & $\begin{array}{l}\text { Pole } \\
{\left[\text { photons }\left(\mathrm{cm}^{2} \mathrm{~s} \mathrm{ster} \mathrm{keV}\right)^{-1}\right]}\end{array}$ & $\begin{array}{l}\text { Ratio } \\
\text { pole/plane }\end{array}$ & $\begin{array}{l}\text { Extrapolation } \\
\text { of } 1-10 \mathrm{keV} \\
\text { Power law (no } \\
\text { interstellar ab- } \\
\text { sorption) [pho- } \\
\text { tons (cm }{ }^{2} \mathrm{~s} \mathrm{ster} \\
\mathrm{keV})^{-1} \text { ] }\end{array}$ \\
\hline $18-24^{\mathrm{a}}$ & $0.69-0.52$ & 50 & 35 & & 18 \\
\hline $44-60^{\mathrm{a}}$ & $0.28-0.21$ & 300 & 120 & 1.4 & 65 \\
\hline $66-90$ & $0.19-0.14$ & $\sim 500$ & $\sim 500^{\mathrm{b}}$ & $\sim 1^{\mathrm{b}}$ & 116 \\
\hline
\end{tabular}

a Intensities are from Davidsen et al. (1972).

b Estimated using the flux at the pole observed by Yentis et al. (1972) and the observation of Gorenstein and Tucker (1972) that the pole/plane ratio is 1.5 in the 44-80 $\AA$ band. Since the 44-60 $\AA$ ratio is about 2.5 , the $66-90 \AA$ ratio must be about 1 to give the $44-80 \AA$ result.

X-rays which originate beyond the SMC. As seen in Table I, this upper limit corresponds roughly to the ratio of the $44 \AA$ flux calculated from an extrapolation of the $1-10 \mathrm{keV}$. power law to the total flux observed toward the poles. This raises the question of whether or not there is any extragalactic contribution to the soft X-ray background beyond that expected from an extrapolation of the power law; we discuss this below.

\subsection{Observational Data}

The currently available data can be summarized for three separate ranges where broad-band measurements have been made within the $0.1-1 \mathrm{keV}$ energy region. Table I gives average values for the flux in the 66-90 $\AA, 44-60 \AA$, and 18-24 $\AA$ regions. In the 66-90 $\AA$ range, only two preliminary results are available (Yentis et al., 1972; Gorenstein and Tucker, 1972). Most of the recent soft X-ray observations have been made in the 44-60 $\AA$ region, and a large fraction of the sky has now been surveyed. Figure 1 shows the Naval Research Laboratory (NRL) results (Davidsen et al., 1972) for the galactic anti-center direction, and Figure 2 shows the Wisconsin results (Bunner et al.. 1972) for the galactic center. These two groups find very similar results in regions where they have both observed. The Lawrence Radiation Laboratory 
(LRL) group (Palmieri et al., 1971) using a somewhat different proportional counter dectector with a considerably thinner window of Formvar, rather than the Kimfol used by the other two groups, find a flux near $b^{\mathrm{II}}=50^{\circ}$ of 270 photons $\left(\mathrm{cm}^{2} \mathrm{~s}\right.$ ster $\mathrm{keV})^{-1}$ which is in reasonable agreement with the other results. However, they also
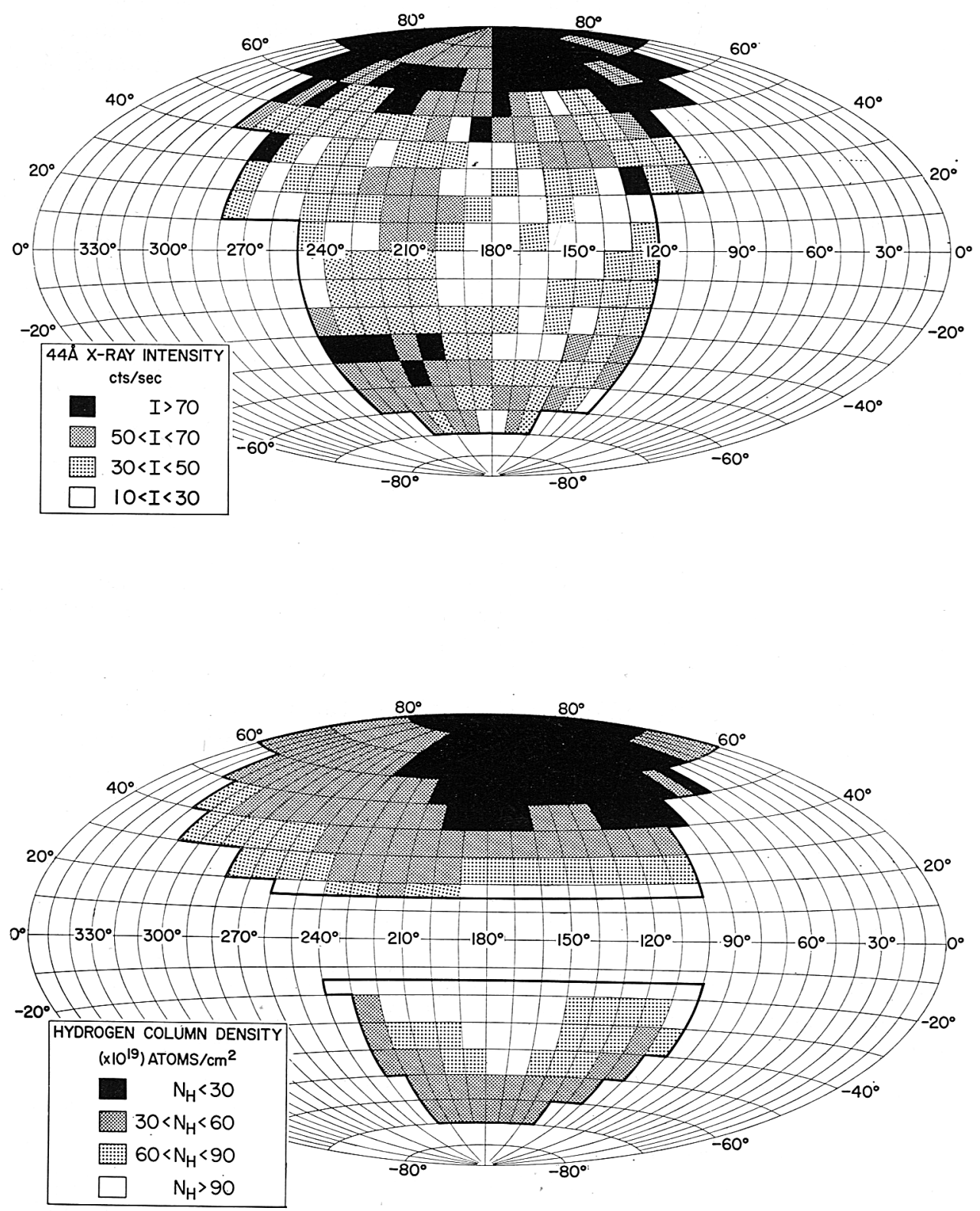

Fig. 1. Top: Map of the sky in the direction of the galactic anti-center in the 44-60 $\AA$ X-ray band. A count rate of $80 \mathrm{~s}^{-1}$ corresponds to a flux of 300 photons $\left(\mathrm{cm}^{2} \mathrm{~s} \text { ster keV) }\right)^{-1}$ (Davidsen et al. 1972). Bottom: Hydrogen $(21 \mathrm{~cm})$ column density of Tolbert(1971). Both maps are in New Galactic Coordinates. 


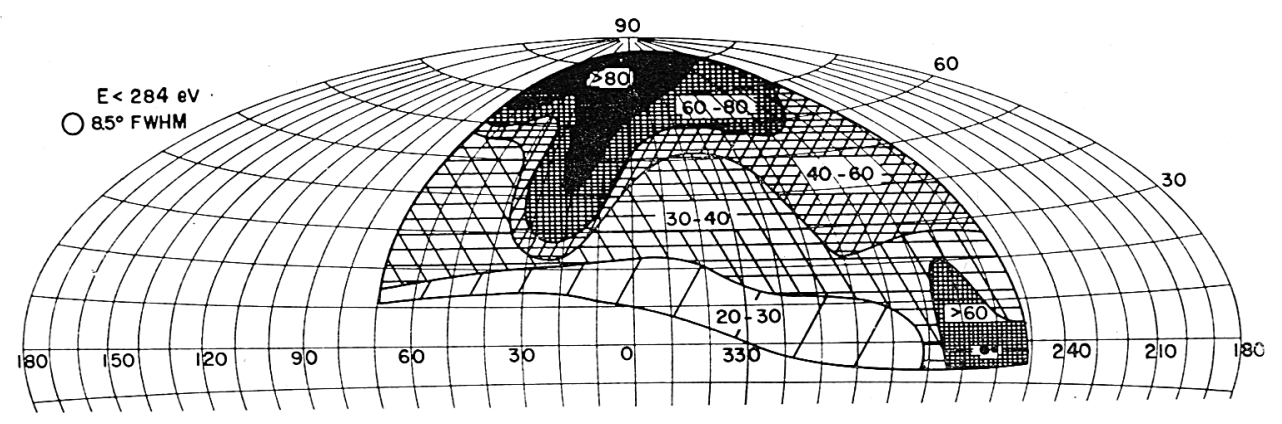

Fig. 2. Map of the sky in the galactic center direction in the 44-60 $\AA$ X-ray band. A count-rate of $20 \mathrm{~s}^{-1}$ corresponds to a flux of 120 photons $\left(\mathrm{cm}^{2} \mathrm{~s}\right.$ ster keV)-1 (Bunner et al., 1972).

find a large flux of 630 photons $\left(\mathrm{cm}^{2} \mathrm{~s} \text { ster } \mathrm{keV}\right)^{-1}$ in a band $l^{\mathrm{II}}=300-340^{\circ}$ near $b^{I I}=20^{\circ}$ which is absent in the Wisconsin results (Figure 2). This discrepancy may be due to low energy particle or UV contamination in the thinner, Formvar window detector.

There is also a considerable amount of data in the region from $0.5-1 \mathrm{keV}$. The NRL group (Henry et al., 1971; Davidsen et al., 1972) has used a Teflon window proportional counter which provides a fluorine $\mathrm{K}$-edge filter with a transmission band from $18-24 \AA$. The peak transmission is only $20 \%$ at $18 \AA$, as opposed to $45 \%$ for Kimfol at $44 \AA$, so that the statistics do not permit a detailed mapping. However, if the counts are put in $10^{\circ}$ galactic latitude bins and summed over all longitudes, the results are statistically significant. These data are shown in Figure 3 (right) along with

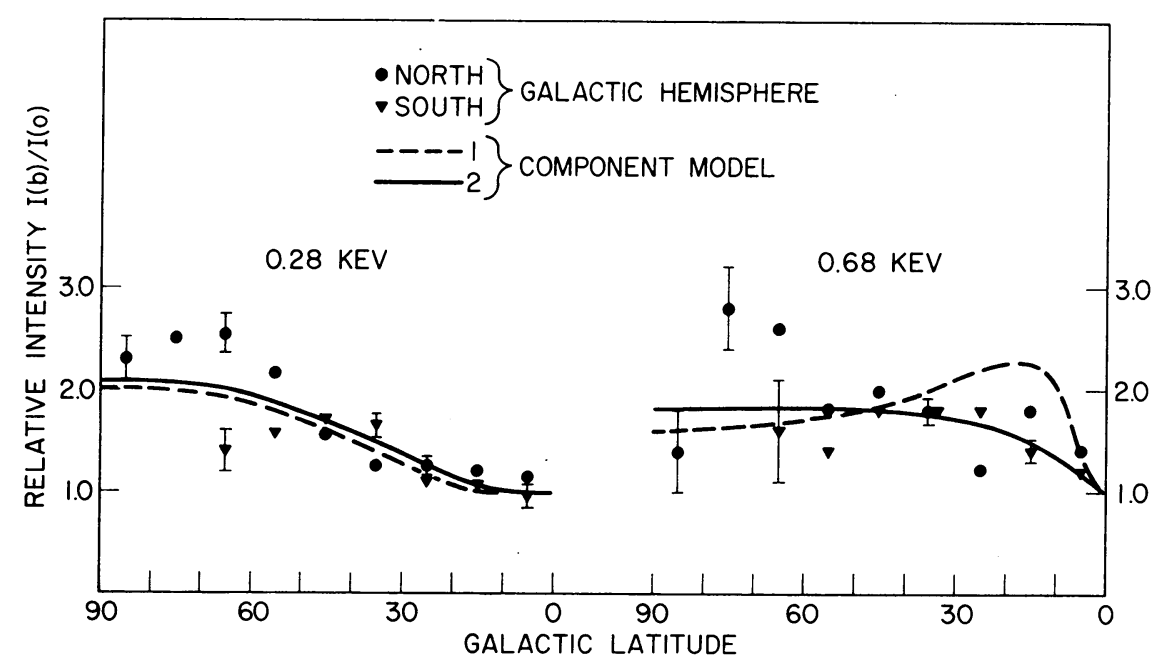

Fig. 3. Latitude dependence of the 44-60 $\AA, 0.28 \mathrm{keV}$ (left), and 18-24 $\AA, 0.68 \mathrm{keV}$ (right), X-ray data. The intensity is normalized to the values in the galactic plane. The dashed and solid curves represent the latitude dependence predicted by the one and two-component models discussed in the text, respectively (Davidsen et al., 1972). 
similar results in the 44-60 $\AA$ band (left). Similar data have been obtained by the Wisconsin and LRL groups using the broader band, $0.5-1 \mathrm{keV}$, provided by the pulse-height resolution of the proportional counter.

\subsection{DisCRETE FEATURES}

In presenting the average soft $\mathrm{X}$-ray fluxes toward the galactic poles and plane, we have ignored several features which are prominent at least in the $44 \AA$ data. In Figure 1, there is an area of enhanced $\mathrm{X}$-ray emission north of the galactic plane near $l^{\mathrm{II}}=210^{\circ}$. The hydrogen column density is anomalously low in this direction and may account for a high transmission of either a galactic or extragalactic flux. A clustering of strong sources could also produce the high intensity, but no strong sources have been reported. In Figure 2, there is a prominent ridge of high intensity which extends from near the North Galactic Pole down towards the plane at $l^{\mathrm{II}}=$ $=0-30^{\circ}$. Bunner et al. (1972) have pointed out that this coincides fairly closely with the North Polar Spur. There is also a high intensity feature in the plane near $l^{\mathrm{II}}=260^{\circ}$ which is associated with the Vela supernova remnant. In spite of these features, however, the soft X-ray background in a large part of the sky has a general dependence on galactic latitude from which it seems possible to deduce fluxes near the pole and in the plane which are more or less independent of galactic longitude.

\subsection{MODELS}

We can use these data to examine two simple models for the origin of the soft X-ray background. The first is a one-component model in which the origin is entirely galactic, with the emission occurring in a disk of scale-height $z_{\mathrm{s}}$. The second is a twocomponent model in which there is both a galactic component and an extragalactic component which is isotropic outside the absorbing layers of the Galaxy. The neutral hydrogen is also assumed to be distributed in a disk with scale-height $z_{H}$. The parameters which can be varied are $z_{\mathrm{s}} / z_{H}$; the absorption cross-section per H-atom, $\sigma$, which is a function of $\mathrm{X}$-ray energy; and, in the case of the two-component model, the ratio of the galactic to extragalactic emission.

The data in Table $I$ indicate a pole/plane ratio of $\sim 1$ in the $66-90 \AA$ region. At these wavelengths, $\sigma$ is large and the $\mathrm{X}$-ray mean free path is small compared with the scale-height of the hydrogen. Therefore, in the one-component model, if $z_{\mathrm{s}} \geqslant z_{\mathrm{H}}$, the 66-90 $\AA$ radiation will appear isotropic thus satisfying the constraints imposed by the data. Likewise, the 44-60 $\AA$ data can be fit by this model if we take $z_{s}>z_{H}$ so that the $\mathrm{X}$-rays appear more intense toward the galactic poles where the optical depth is about unity. This explanation for the latitude dependence of the 44-60 $\AA$ radiation has been suggested recently by a number of authors. A detailed calculation with $z_{\mathrm{s}} / z_{H} \simeq 5$ gives the fit shown by the dashed line in Figure 3 (left). However, the 18-24 $\AA$ data cannot be fit any simple way with this model. The optical depth toward the galactic poles is only about 0.15 so that the $\mathrm{X}$-ray intensity should first increase with decreasing galactic latitude before it decreases near the plane where the optical depth becomes larger than unity. A detailed calculation for the one-component 
model gives the dashed curve in Figure 3 (right). The data do not seem to indicate a peak intensity at low to intermediate latitudes. This problem in fitting the latitude dependence at wavelengths shorter than $44 \AA$ was also noted by the Wisconsin group (Bunner et al., 1969) in attempting to fit their data in an energy region from 0.5-1.0 $\mathrm{keV}$.

The two-component model avoids this problem with the 18-24 $\AA$ data by allowing us to keep $z_{\mathrm{s}} \simeq z_{\boldsymbol{H}}$. With this constraint, the sum of the galactic and extragalactic components can be made to decrease monotonically with decreasing latitude in the 18-24 $\AA$ region. The results of detailed calculations for both the 44-60 $\AA$ and the 18-24 $\AA$ data are shown by the solid curves in Figure 3 . The model predicts that the 44-60 A radiation observed toward the galactic poles is approximately $\frac{2}{3}$ extragalactic and $\frac{1}{3}$ galactic. The $66-90 \AA$ radiation will still appear isotropic, however, since the mean free path is still less than the neutral hydrogen scale-height, and all but a tiny fraction of the extragalactic component will be absorbed out.

It appears, therefore, that while the 44-60 $\AA$ and $66-90 \AA$ observations could be explained by a totally galactic origin for the soft X-rays, the 18-24 $\AA$ flux is more naturally explained by a combination of galactic and extragalactic emission. This same model can also provide the flux observed in the two longer wavelength bands as it must if we wish to keep the same mechanisms and source distributions for all the soft $\mathrm{X}$-ray emission. The hypothesized extragalactic component must be examined, however, in the light of the negative result for absorption of the background radiation by the Small Magellanic Cloud (McCammon et al., 1971). As mentioned previously, an upper limit of $25 \%$ was set on the fraction of the radiation in the 44-60 $\AA$ band which originates beyond the SMC. From our two-component model we find that $67 \%$ of the observed X-rays should be extragalactic in origin in the direction of the galactic poles where the optical depth is about unity.

There are several ways in which these observations might be reconciled. The SMC is at galactic latitude, $b^{\mathrm{II}} \simeq-40^{\circ}$, considerably away from the South Galactic Pole At this latitude, the two-component model then predicts that only $50 \%$ of the radiation is extragalactic in origin. This value is somewhat closer to the upper limit quoted by the Wisconsin group (McCammon et al., 1971). Another possibility is that there is soft X-ray emission in the SMC itself which fills in for the extragalactic X-rays which have been absorbed. The Wisconsin group points out that the only known source in the SMC which is observed in the $2-6 \mathrm{keV}$ energy range does not appear to provide enough soft $\mathrm{X}$-ray photons to compensate for the absorption. However, in our own galaxy we know that there is soft $X$-ray emission which is not associated with any known sources of $\mathrm{X}$-rays with energies above $1 \mathrm{keV}$. In fact, most of the galactic component in our two-component model is exactly such soft $\mathrm{X}$-ray emission. if we attribute a similar source of soft $\mathrm{X}$-rays to the SMC, we find that the emission could compensate for about one-half of the absorbed X-rays so that the fraction of extragalactic X-rays in these observations will be reduced to $50 \%$, or less, of the total flux.

These considerations introduce some uncertainty into the interpretation of the SMC observation, and allow an origin beyond the SMC for a sizeable fraction of the 
soft X-ray flux. Even if none of the soft X-ray flux originates beyond the SMC, a two-component model could still be a good approximation with a large spherical galactic halo source replacing the extragalactic component. The emission would still be isotropic, and the calculational details of the model would remain unchanged. Using data from the same flight as the SMC observation, McCammon et al. (1971) also rule out the existence of a substantial background contribution from such a hot galactic halo because they see no correlation between changes in the hydrogen column density and the $44 \AA \mathrm{X}$-ray intensity. However, the interpretation of this observation relies heavily on the accuracy of the $21-\mathrm{cm}$ data. The survey data of McGee et al. (1966), which was used by the Wisconsin group, may not be reliable at the low hydrogen column densities $\left(1-2 \times 10^{20} \mathrm{~cm}^{-2}\right)$ in the observed direction. In contrast to the Wisconsin observation, Davidsen et al. (1972) do find a correlation at high galactic latitudes over a large area of the sky between the hydrogen column density and the $44 \AA$ intensity.

\section{Theories of the Origin of the Soft X-Ray Background}

The low energy $\mathrm{X}$-ray background (0.1-10 keV) may be the integrated effect of large numbers of discrete $\mathrm{X}$-ray emitters, as discussed in Section 1, or it may be due to processes occuring in the reaches of intergalactic, interstellar, or even interplanetary space. The $\mathrm{X}$-ray and $\gamma$-ray background spectrum is considerably more complex than that of any single discrete source that has been observed, suggesting that many processes may contribute to the observed total background. We first summarize those theories that involve a diffuse emission mechanism, and then consider models for the $0.1-1 \mathrm{keV}$ galactic emission. An extensive review of extragalactic mechanisms has been published by Silk (1970).

\subsection{Diffuse EMISSION SOURCES}

Diffuse emission sources involve either non-thermal or thermal mechanisms for the production of the radiation. Non-thermal mechanisms produce $\mathrm{X}$-rays through the interaction of high energy protons or electrons with electromagnetic radiation, magnetic fields, or other particles. Sources of the high energy particles which have been proposed include radio galaxies, 'normal' galaxies, galactic nuclei, and neutron stars in galaxies. Difficulties with such mechanisms center on inadequate primary particles, insufficient target material, and inefficient energy conversion. On the other hand, theories of thermal emission have difficulty in accounting for the heating of the proposed gas to the high temperatures required, and difficulty in ionizing the gas in the time available.

The most carefully studied non-thermal mechanism is the inverse Compton effect, in which high energy electrons from strong radio galaxies (Felten and Morrison, 1966) or from 'normal' radio galaxies (Brecher and Morrison, 1969) scatter from photons of the $3 \mathrm{~K}$ microwave background radiation and produce $\mathrm{X}$-rays. Clearly, this interaction must occur; the only question is whether the bulk of the $\mathrm{X}$-ray background 
could be so produced. Cowsik (1971) has argued that the spectral form of the 2-1000 $\mathrm{keV}$ background is not compatible with this theory for its origin; he suggests, however, that the radiation observed below $2 \mathrm{keV}$ and above $1000 \mathrm{keV}$ might be (at least partly) produced by the inverse Compton mechanism.

\subsection{THERMAL MECHANISMS}

The thermal mechanism of radiation from a hot intergalactic gas was described by Field and Henry (1964) and proposed by Henry et al. (1968) as the source of the background radiation observed at $44 \AA$. In the 'big bang' cosmology, however, nonthermal components such as high energy particles are also required to ionize and heat the intergalactic gas. These particles (protons and/or electrons) will lose part of their energy through non-thermal bremsstrahlung in collisions with thermal electrons and, therefore, the spectrum produced will be modified from a pure thermal spectrum. The inefficiency of the non-thermal bremsstrahlung, however, probably ensures that this effect will be relatively small.

Arons (1971) has examined the ionization process on the assumption that the flux of ionizing radiation is produced by quasars, and that the quasars are at their cosmological distance and had a very high space density in early times. He concludes that in the case of a high-density intergalactic medium, the zones ionized by the quasars never join up. This would mean that broad Lyman- $\alpha$ absorption troughs should appear in the red shifted ultraviolet spectra of quasars, contrary to observation. However, a lower-density medium (up to $\frac{1}{3}$ closure density) can become fully ionized.

It should eventually be possible to obtain more information on the efficacy of quasars as ionizers of the intergalactic gas by observing indirectly the particle emissions from nearby (recent) quasars, on the assumption that these are not very different from the earlier quasars. Byram et al. (1970), Bowyer et al. (1970), and Kellogg et al. (1971a) have detected X-rays from the quasar 3C 273. If the X-rays are produced by relativistic particles, it might be possible to determine the rate of emission and spectrum of the particles through careful study of the spectrum and spatial distribution of the X-ray emission.

If an ionized intergalactic gas does exist, it may be at a temperature of $\sim 300 \times 10^{6} \mathrm{~K}$ (Cowsik, 1971) or at $\sim 3 \times 10^{6} \mathrm{~K}$ (Henry et al., 1968). The higher temperature gas would be used to explain the greater part of the $\mathrm{X}$-ray spectrum, while the lower temperature gas would be used only to explain the excess observed below $1 \mathrm{keV}$. These alternatives permitted by the observations result in very different relationships between the diffuse and condensed matter in the universe. The condensed matter (galaxies) is distributed in a distinctly non-uniform manner (Limber, 1954). If the ionized matter is at only $3 \times 10^{6} \mathrm{~K}$, it must respond gravitationally to the presence of these irregularities. In particular, it will tend to fall into clusters of galaxies (Gott and Gunn, 1971). It will, therefore, be clumped at the present time, and a smaller total quantity will be required to account for the observed $44 \AA \mathrm{X}$-ray emission. If, on the other hand, the gas is at $300 \times 10^{6} \mathrm{~K}$ it would escape freely from clusters of galaxies 
and presumably would be uniformly distributed. A density of $3 \times 10^{-6}$ particles $\mathrm{cm}^{-3}$ would be needed to account for the emission, probably sufficient to close the universe. The $2-100 \mathrm{keV}$ background radiation is isotropic to within a few percent (Schwartz et al., 1970) arguing consistency with a $300 \times 10^{-6} \mathrm{~K}$ plasma. Angular variations at a level of a few percent, reported recently (Murray et al., 1971), could indicate that an extremely hot gas is not the origin of the radiation, or could simply indicate that a second, highly irregular $\mathrm{X}$-ray component is involved.

\subsection{Clusters of galaxies}

Observations of the relationship of the X-ray background to clusters of galaxies are clearly important. Meekins et al. (1971) detected a flux of X-rays from the direction of the Coma cluster of galaxies. Gursky et al. (1971) showed that the $\mathrm{X}$-ray emission comes from nearly the position of the optical cluster center, and that the source is extended. It is not possible to distinguish between a thermal and a power-law spectrum, but if the radiation is interpreted as thermal, a temperature of $\sim 70 \times 10^{6} \mathrm{~K}$ is implied. The intensity of the radiation is so low that there cannot be enough matter present in the form of gas at this temperature to gravitationally bind the cluster. Can the additional 'missing mass' be present in the form of gas at lower temperature? The upper limit of Meekins et al. at $44 \AA(0.28 \mathrm{keV})$ rules out

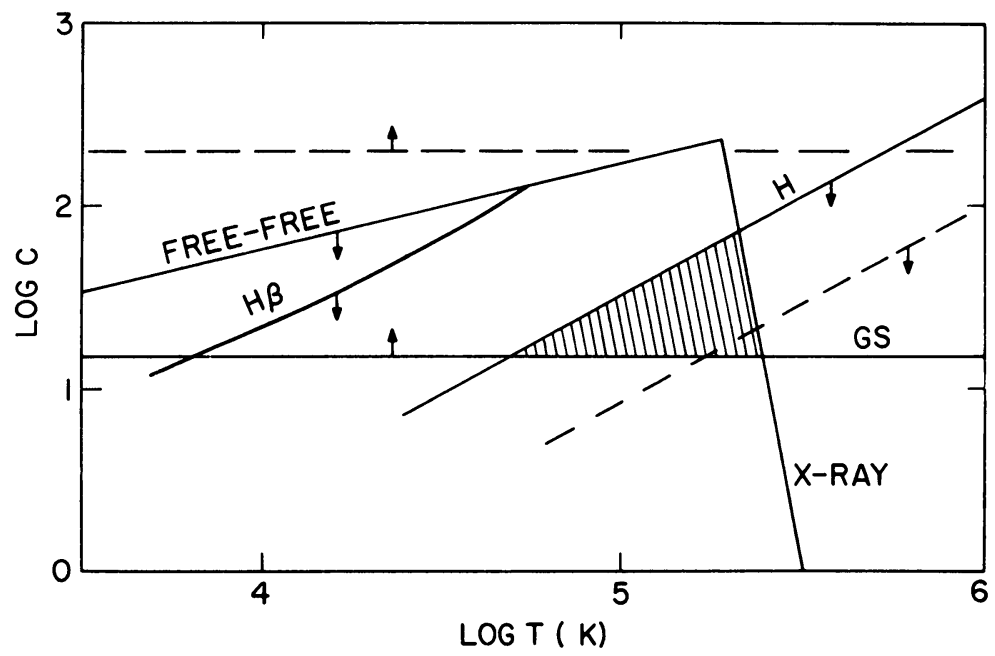

Fig. 4. The logarithm of $C(=1 / f)$, where $f$ is the fraction of the Coma cluster of galaxies occupied by hot clouds of gas, is plotted against the logarithm of the temperature. Observations and theoretical considerations limit the regions of the plane that might contain the point representing the actual condition of the Coma cluster, if the Hubble constant is $50 \mathrm{~km} \mathrm{~s}^{-1} \mathrm{Mpc}^{-1}$ and if the cluster contains a 'binding' mass of ionized gas. In particular, Woolf (1967) set the free-free emission and H $\beta$ limits shown, while Meekins et al. (1971) set the X-ray limit. Henry (1972) set the L $\alpha$ emission limit shown (line labelled $\mathbf{H}$, the result of a more detailed calculation of his original (parallel dashed line) model), while the limit due to Goldsmith and Silk (1972) is the horizontal solid (GS) line (revision of their original, 1971, limit). The hatched area is the surviving region on the plane that could represent the actual state of a gravitationally-binding mass of ionized gas. 
the possibility of sufficient quantities of gas at $\sim 5 \times 10^{5} \mathrm{~K}$, but if a general intergalactic medium exists at the latter temperature, one could consider a situation in which matter falls gravitationally into the Coma cluster over a time period of $10^{9} \mathrm{yr}$, and then collapses into clouds in the temperature range $10^{4}$ to $10^{6} \mathrm{~K}$. (Cooler clouds are ruled out from the lack of $21-\mathrm{cm}$ absorption or emission.) Motion of these clouds, and of the galaxies, heats a small amount of residual gas to $70 \times 10^{6} \mathrm{~K}$. Goldsmith and Silk (1972) have studied such a cloud model, and suggested its $L \alpha$ recombination radiation as a means of detecting it. Henry (1972) set an upper limit to the redshifted $\mathrm{L} \alpha$ flux from the Coma cluster of galaxies. His result is summarized in Figure 4. That limit, with a theoretical limit on the degree of clumping of the gas in the cluster by Goldsmith and Silk (1971), led to the virtual exclusion of the possibility of the presence of a binding mass of hot gas in the cluster. However, Goldsmith and Silk (1972) have revised their theoretical limit. Also, we have done a detailed numerical integration of the Coma cluster density model of Omer et al. (1965), resulting in the revised meaning of the $L \alpha$ limit shown in Figure 4 . The result suggests that if a binding mass of gas exists, it is in the form of clouds at about $2 \times 10^{5} \mathrm{~K}$ which occupy about $6 \%$ of the volume of the cluster. Clearly, further intensive examination of the Coma cluster of galaxies in all wavelength ranges is desirable.

The problem of heating a dense intergalactic medium to $3 \times 10^{6} \mathrm{~K}$ is severe, and the problem is clearly more difficult in the case of the higher temperature model. In the latter case, the energy for heating could not come from nuclear energy sources. Gravitational collapse can provide arbitrarily large amounts of energy in theory, but putting that energy in the right place - the low-density regions of intergalactic space - is very difficult. The problem may be avoided by adopting a steady-state origin, but this is not a very satisfying solution.

\subsection{LOW ENERGY GALACTIC X-RAYS}

No matter how the problem of the origin of the extragalactic component is resolved, it is clear that some galactic component is present at low energies $(0.1-1 \mathrm{keV})$. This galactic emission is rather smoothly distributed and the production mechanism (or mechanisms) is not understood. Many origins have been proposed, most of which involve discrete sources. These models can be divided into those in which the sources have a larger scale-height than the neutral hydrogen and those in which the scaleheight is the same as the hydrogen. These correspond to the galactic components required by the one- and two-component models, respectively, which were discussed in the previous section.

The large scale-height models include proposals for soft $\mathrm{X}$-ray emission from a hot corona surrounding white dwarfs (Strittmatter et al., 1972) and from flare stars (Cavallo and Horstman, 1972) where the emission is presumably due to a hot stellar atmosphere which also produces the observed optical and radio flares. The models which have scale-heights comparable to the neutral hydrogen include soft $\mathrm{X}$-rays from supernova remnants (Ilovaisky and Ryter, 1971) and accretion of interstellar matter by defunct pulsars (Ostriker et al., 1970). The supernova remnant model 
suffers from the problem that the number of such sources is small and, therefore the average distance is great. The supernova remnants only contribute at galactic latitudes within a few degrees of the plane and must have very large soft $X$-ray luminosities to overcome interstellar absorption. The smoothness of the soft $\mathrm{X}$-ray distribution may already rule out such a low source density model. The defunct pulsar model, on the other hand, produces a high density of sources if one accepts the exponential decay of the neutron star birth rate assumed by the authors.

Another class of models which could produce the galactic soft X-ray component is nearby diffuse sources. In particular, Shklovski and Sheffer (1971) have proposed that the galactic spurs, which they assume are old nearby supernova remnants, are such diffuse soft $\mathrm{X}$-ray sources. They propose that the spurs, because they are nearby, provide the source of emission in the direction of the galactic poles while the integral effect of old type II supernova remnants accounts for the emission in the plane. Recently, the North Polar Spur has been observed to be a source of soft X-rays (Bunner et al., 1972). However, other spur structures such as Loop III and parts of other loop structures were observed by Davidsen et al. (1972) and not found to be X-ray emitters with an upper limit significantly below that of the North Polar Spur. It appears, then, that the spurs are not generally soft X-ray sources of sufficient intensity to account for a large fraction of the background.

One other possible galactic source of soft X-rays that was mentioned in the preceding section is the galactic halo proposed by Spitzer (1956). However, even in his highest temperature model $\left(10^{6} \mathrm{~K}\right)$, where the electron density is $5 \times 10^{-4} \mathrm{~cm}^{-3}$ and the radius is $8 \mathrm{kpc}$, there is insufficient soft $\mathrm{X}$-ray emission to explain the observed background. The crude spectral observations seem to require a slightly higher temperature of $3 \times 10^{6} \mathrm{~K}$ and an emission measure, $\int n_{e}^{2} \mathrm{~d} l$, of about $10^{-1} \mathrm{pc} \mathrm{cm}^{-6}$. If one is to maintain Spitzer's assumption of static pressure equilibrium at the interface between the halo gas and the neutral hydrogen disk, then the temperature and emission measure can be used to determine the radius of the halo. For the parameters which best fit the observations, the radius is $10^{6} \mathrm{pc}$. Therefore, the halo is not really associated with the Galaxy alone and, in fact, would not be gravitationally bound. Instead, it is really an intracluster gas, and the distinction between galactic and extragalactic becomes a semantic one. Burke and Hartwick (1972) have recently suggested, however, that one could give up the assumption of static pressure equilibrium and still support a local halo with the required temperature and emission measure by means of a galactic wind mechanism. These authors propose that an extended X-ray source recently observed near the galactic center (Kellogg et al., 1971b) may be evidence for a galactic nucleus with a temperature of more than $10^{7} \mathrm{~K}$ and densities of $0.1 \mathrm{~cm}^{-3}$ which would drive the galactic wind.

\section{Appendix}

OBSERVING TECHNIQUES

Observations of the soft $\mathrm{X}$-ray background radiation require the detection of a small 
flux of photons of rather low energy in the presence of substantial interference from non-X-ray events in the detector. Further difficulties are encountered when the weak photon flux is subdivided in terms of energy to form a spectrum. In addition, conventional astronomical techniques are difficult to apply in the X-ray region: polished surfaces reflect X-rays only at small angles, solid materails transmit soft X-rays only for very short distances $(\approx 0.1 \mathrm{~mm})$, photographic film is relatively insensitive, and so on. In fact, detection of the soft X-ray background has been achieved almost exclusively by means of the photoionization of gases.

\section{Proportional counter detectors}

In a typical gas-filled X-ray detector, a window serves both to transmit the X-rays and to contain the gas; a high electric field in the gas volume causes avalanche multiplication of the original photoelectrons. The multiplication is necessary because, for example, a $1 \mathrm{keV}$ photon can produce only about 36 ion pairs in the detector gas, and such a small signal would not be electronically detectable. With a gas multiplication of $10^{4}$ to $10^{5}$, each photon event is easily detectable, even down to energies $\sim 0.1 \mathrm{keV}$, since the equivalent noise in the amplifier can be kept below $10^{4}$ electrons. In the earlier days of X-ray astronomy, the gas-filled detectors were operated at much higher gains $\left(\sim 10^{8}\right)$, in the saturated avalanche or Geiger counter mode, but it was soon realized that the lower gain detectors (proportional counters) were almost as simple to build and offered the great advantage of some spectral resolution (by analyzing the heights of the pulses).

Since the gas multiplication in the detector is nearly constant for all events, the spectral resolution can be approximately determined by considering the Poisson statistics of the number of original ion pairs. For monoenergetic photons at $1 \mathrm{keV}$, the average of $\sim 36$ ion pairs produced has $\sigma \simeq \sqrt{ } 36=6$; the pulse height analysis of such $1 \mathrm{keV}$ line emission would show an approximately Gaussian distribution whose full width at half maximum (FWHM) would be $\sim 2.35 \sigma=14$ (in units of ion pairs). Then the percentage FWHM for $1 \mathrm{keV}$ would be $\sim \frac{14}{35}=40 \%$; this corresponds closely to the measured value. Figure 5 illustrates the efficiency and resolving power of a typical proportional counter. The great advantage of the gas-filled proportional counter over other possible photoelectric detectors for soft X-rays is that it provides a crude photon spectrum, and it can be scaled up to almost any size (currently in the thousands of $\mathrm{cm}^{2}$ ) with virtually no degradation of the spectral resolution.

\section{FIELD OF VIEW}

For studies of the soft X-ray background, the small flux of X-rays necessitates a large field of view (as well as a large collecting area) to improve the statistical precision of the measurements. However, the large and increasing number of known point sources, as well as the spatial variations in the background flux itself that are now becoming apparent, require a small field of view. The majority of observations to date have used mechanical baffles to define the field of view, which is generally in the range $10-200 \mathrm{deg}^{2}$; collimation in one direction has been as fine as $\sim 1^{\circ}$ and as large as $\sim 45^{\circ}$. 


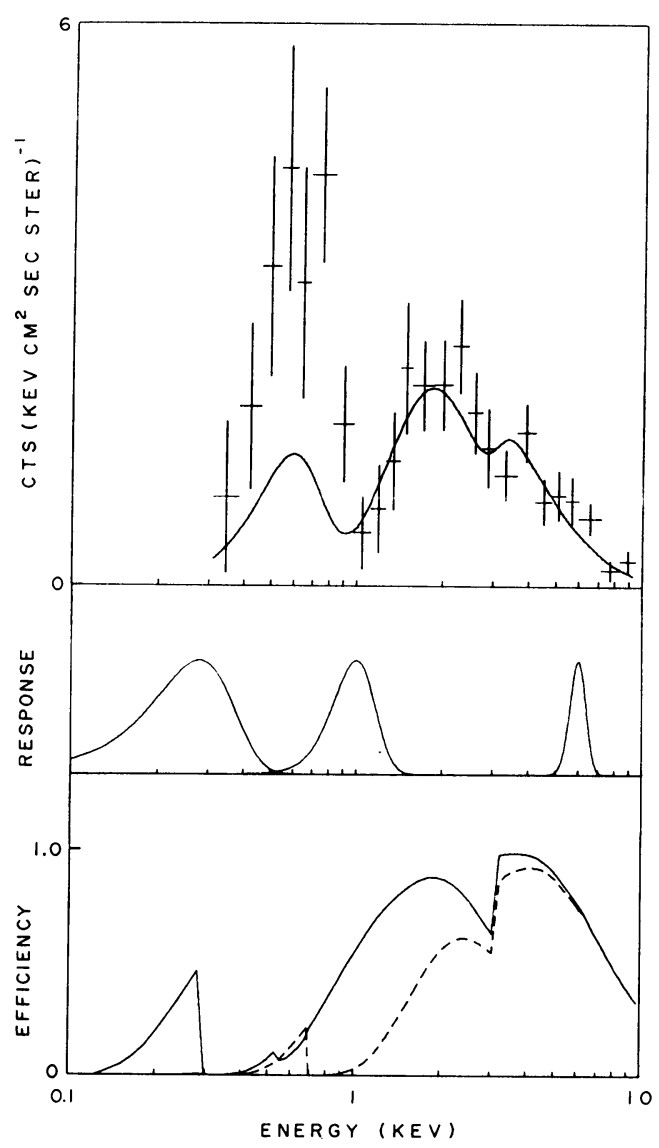

Fig. 5. Spectral resolution of a gas-filled proportional counter. The abscissa for all curves is energy in units of $\mathrm{keV}$. At the bottom of the figure is shown the detection efficiency of argon-filled counters with $2 \mu$ polycarbonate window (solid curve) and $3.2 \mu$ Teflon window (dashed curve). The center section shows the response these detectors would have to line emission at $0.28,1.0$ and $6.0 \mathrm{keV}$. In the upper part of the figure, data obtained near the galactic pole with the Teflon window counter are plotted together with the theoretical response of the counter to a simple power-law spectrum (solid curve) which fits the data above $1 \mathrm{keV}$; the low-energy excess in the data is clearly apparent.

$\mathrm{X}$-ray telescopes are useful only below $1 \mathrm{keV}$, because the reflectance drops sharply at higher energies; even below $1 \mathrm{keV}$, the field of view is restricted by the reflection angles to $\sim 5^{\circ}$. A further complication with the telescopes is the fact that the efficiency of the overall instrument varies both with wavelength and with angle of arrival of the incoming photons. Proportional counters with simple collimators may have some small reflection effects themselves at the longest wavelengths $(0.1-0.3 \mathrm{keV})$, but generally the efficiency at different angles and wavelengths is easily calculable from the geometry of the collimator and the mass absorption coefficients of the window and gas materials. 


\section{THE NON-X-RAY BACKGROUND}

Proportional counters are sensitive to any source of ionization that deposits an energy in the counter gas equivalent to the X-ray energy being observed $(0.1-10 \mathrm{keV})$. The main components of this non-X-ray background in the detector are cosmic rays, soft electrons trapped by the Earth's magnetic field, $\gamma$-rays that scatter in the detector, the natural radioactivity of materials in the detector assembly, and other minor sources of particles (such as those associated with solar activity). It is worth noting that other types of photoionization or photoelectric detectors for X-rays, such as X-ray photocathodes, are considerably more susceptible to these interfering effects than the proportional counter because even events outside the range of X-ray energies are counted in such devices.

The techniques used to eliminate the non-X-ray background include the use of coincidence counters (also called guard or veto detectors) surrounding the X-ray detector, discrimination based on the risetimes of the electrical pulses in the proportional counters, and the use of X-ray telescopes to collect the X-rays on a small area detector. Risetime analysis depends upon the fact that the electrons liberated in the counter gas by the incoming X-ray are all collected nearly simultaneously, while the ionization produced by a particle track in the gas is collected over a longer time. Unfortunately, this difference disappears at energies below a few $\mathrm{keV}$, and the technique is efficient only above $3 \mathrm{keV}$. Focusing X-ray collectors reduce the non-X-ray background in two ways: first, by reducing the size of the detector relative to the effective area of the total instrument and, second, by shielding the detector against direct paths of soft electrons that could penetrate the counter window.

Coincidence counters around the central X-ray detector have taken almost every conceivable form. Some improvement can be made by placing the coincidence counters in the same gas volume as the X-ray counter, separated only by wires or screens. This arrangement has the advantages that (1) low energy particles are not stopped by the walls of the X-ray counter before reaching the veto counters, and (2) photon scattering effects in the walls are minimized. While this technique is quite effective for penetrating particles, it suffers from the drawback that only 5 sides of the X-ray counter can be protected. In the soft X-ray region the detector windows are already so thin that there is no possibility of placing a veto counter in front of the window.

Another source of non-X-ray background is the ultraviolet sensitivity of the X-ray detectors. The problem exists because the inner surfaces (generally metallic) of the gas-filled detectors do not have a negligible photoelectric yield; the small pulses from UV photons pile up so as to mimic low energy X-ray events. Most groups have taken steps to desensitize the counter walls and have made measurements to verify that the UV response is sufficiently low.

\section{SPECTROSCOPY OF THE SOFT X-RAY BACKGROUND}

Although several well-established techniques exist for producing high resolution $\mathrm{X}$-ray spectra (notably use of crystals and gratings), the difficulty of applying them to 
the soft X-ray background is the requirement for a nearly parallel beam, which thus limits the field of view, and therefore the flux, to quite low values. This factor, combined with the small collecting areas typical of such devices, means that such spectroscopy of the X-ray background is not likely to be done in the near future.

In the meantime, a considerable effort has gone into a crude form of spectroscopy using pulse height analysis in proportional counters and 'filter photometry' based upon the absorption edges in the detector window material (or occasionally in a separate filter). In fact, the strongest evidence that the background fluxes below $1 \mathrm{keV}$ are true X-rays is that the pulse height spectra show the characteristic peaks of the window elements (usually carbon or fluorine). Of particular importance is the 'peak-to-valley' ratio at these low energies, since the interfering effects of UV response, soft electron fluxes, and general system noise tend to be smoothly varying functions of photon energy; the peaking of the spectrum is thus characteristic of true $\mathrm{X}$-rays transmitted through the window. This effect is shown clearly in Figure 5 (top) where the peak near $0.6 \mathrm{keV}$ is due to the fluorine edge of the Teflon window detector.

Above $1 \mathrm{keV}$ the detector response is generally much smoother (also shown in Figure 5), and here the problem is to obtain accurate wavelength and efficiency calibrations so that the X-ray background spectrum can be unfolded with some precision. In practice, the intrinsic background spectrum cannot be easily unfolded from the pulse height spectrum of the detector. A reverse process is normally used in which a trial source spectrum is folded with the known parameters of the detector (such as efficiency, resolution, non-linearity, etc.); the resulting theoretical curve is then compared with the observational data (as shown in Figure 5, top), and the trial spectrum is modified until a satisfactory fit is obtained.

The difficulty in obtaining accurate spectral information with coarse resolution devices and such fitting techniques is illustrated by recent efforts to study spectrum details in the 1-10 keV energy range. Ducros et al. (1970) and Shulman et al. (1971) have reported possible emission features in their diffuse X-ray background data at $5 \mathrm{keV}$ and $7 \mathrm{keV}$, respectively. The $7 \mathrm{keV}$ peak appears weakly in the data of Figure 5 (top), which shows only part of the counts actually obtained. Boldt et al. (1971) and Toor et al. (1972), however, find no evidence for such features and place upper limits that are an order of magnitude smaller than the reported features which were equal to the integrated continuum in the same energy region. Toor et al. (1972) obtained their negative results by using detectors with different fields of view and extracting that part which scales with the solid angle. The fact that no line feature was observed indicates that the original results may have been due to particle interference, which is independent of the collimator field of view. The problem with this interpretation is that it requires equal amounts of X-ray and non-X-ray background in the detectors, whereas earth-looking data and data taken before the rocket doors were opened suggest that the non-X-ray background was much lower. If particle contamination should turn out to be the cause of these anomalies, it may also affect the interpretation of spectral index changes in the $1-20 \mathrm{keV}$ energy range. A greater effort is needed to understand the origin of these discrepancies. 


\section{References}

Arons, J.: 1971, 136th AAS Meeting (San Juan).

Boldt, E. A., Desai, U. D., and Holt, S. S.: 1969, Nature 224, 677.

Boldt, E. A., Desai, U. D., Holt, S. S., and Serlemitsos, P. J.: 1971, Astrophys. J. Letters 167, L1.

Bowyer, C. S., Field, G. B., and Mack, J. E.: 1968, Nature 217, 32.

Bowyer, C. S., Lampton, M., Mack, J., and de Mendonca, F.: 1970, Astrophys. J. Letters 161, L1.

Bradt, H., Naranan, S., Rappaport, S., Zwicky, F., Ogelman, H., and Boldt, E.: 1968, Nature 218, 856.

Brecher, K. and Morrison, P.: 1969, Phys. Rev. Letters 23, 802.

Bunner, A. N., Coleman, P. L., Kraushaar, W. L., McCammon, D., Palmieri, T. M., Shilepsky, A., and Ulmer, M.: 1969, Nature 223, 1222.

Bunner, A. N., Coleman, P. L., Kraushaar, W. L., and McCammon, D.: 1971, Astrophys. J. Letters 167, L3.

Bunner, A. N., Coleman, P. L., Kraushaar, W. L., and McCammon, D.: 1972, Astrophys. J. Letters 172, L67.

Burke, J. A. and Hartwick, F. D. A.: 1972, Nature Phys. Sci. 236, 4.

Byram, E. T., Chubb, T. A., and Friedman, H.: 1970, Science 169, 366.

Cavallo, G. and Horstman, H.: 1972, Nature Phys. Sci. 235, 110.

Cowsik, R.: 1971, 136th AAS Meeting (San Juan).

Davidsen, A., Shulman, S., Fritz, G., Meekins, J. F., Henry, R. C., and Friedman, H.: 1972, Astrophys. J. 177 (in press).

Ducros, G., Ducros, R., Rocchia, R., and Tarrius, A.: 1970, Astron. Astrophys. 7, 162.

Fabian, A. C. and Sanford, P. W.: 1971, Nature Phys. Sci. 231, 52.

Felten, J. E. and Morrison, P.: 1966, Astrophys. J. 146686.

Field, G. B. and Henry, R. C.: 1964, Astrophys. J. 140, 1002.

Friedman, H., Byram, E. T., and Chubb, T. A.: 1967, Science 156, 374.

Goldsmith, D. and Silk, J.: 1971, 136th AAS Meeting (San Juan).

Goldsmith, D. and Silk, J.: 1972, Astrophys. J. 172, 563.

Gorenstein, P. and Tucker, W. H.: 1972, Astrophys. J. 176, 333.

Gott, J. R., III and Gunn, J. E.: 1971, Astrophys. J. Letters 169, L13.

Gunn, J. E. and Ostriker, J. P.: 1969, Astrophys. J. 157, 1395.

Gursky, H., Kellogg, E., Murray, S., Leong, C., Tananbaum, H., and Giacconi, R.: 1971, Astrophys. J. Letters 167, L81.

Hayakawa, S., Kato, T., Makino, F., Ogawa, H., Tanaka, Y., Yamashita, K., Matsuoka, M., Miyamoto, S., Oda, M., and Ogawara, Y.: 1971, Astrophys. Space Sci. 12, 104.

Henry, R. C., Fritz, G., Meekins, J. F., Friedman, H., and Byram, E. T.: 1968, Astrophys. J. Letters 153, L11.

Henry, R. C., Fritz, G., Meekins, J. F., Chubb, T., and Friedman, H.: 1971, Astrophys. J. Letters 163, L73.

Henry, R. C.: 1972, Astrophys. J. Letters 172, L97.

Ilovaisky, S. A. and Ryter, Ch.: 1971, Astron. Astrophys. 15, 224.

Kellogg, E., Gursky, H., Leong, C., Schreier, E., Tananbaum, H., and Giacconi, R.: 1971a, Astrophys. J. Letters 165, L49.

Kellogg, E., Gursky, H., Murray, S., Tananbaum, H., and Giacconi, R.: 1971b, Astrophys. J. Letters 169, L99.

Limber, N.: 1954, Astrophys. J. 119, 655.

Mark, H., Price, R. E., Rodrigues, R. M., Seward, F. D., and Swift, C. D.: 1969, Astrophys. J. Letters 155, L143.

McCammon, D., Bunner, A. N., Coleman, P. L., and Kraushaar, W. L.: 1971, Astrophys. J. Letters 168, L33.

McGee, R. X., Milton, J. A., and Wolfe, W.: 1966, Australian J. Phys. Suppl. No. 1.

Meekins, J. F., Fritz, G., Chubb, T. A., Friedman, H., and Henry, R. C.: 1971, Nature 231, 107.

Murray, S., Tananbaum, H., Matilsky, T., Kellogg, E., Gursky, H., and Giacconi, R.: 1971, 136th AAS Meeting (San Juan).

Omer, G. C., Page, T. L., and Wilson, A. G.: 1965, Astron. J. 70, 440. 
Ostriker, J. P., Rees, M. J., and Silk, J.: 1970, Astrophys. Letters 6, 179.

Palmieri, T. M., Burginyon, G. A., Grader, R. J., Hill, R. W., Seward, F. D., and Stoering, J. P.: 1971, Astrophys. J. 169, 33.

Schwartz, D., Hudson, H. S., and Peterson, L. E.: 1970, Astrophys. J. 162, 431.

Schwartz, D. A., Boldt, E. A., Holt, S. S., Serlemitsos, P. J., and Bleach, R. D.: 1971, Nature Phys. Sci. 233, 110.

Shklovski, I. S. and Sheffer, E. K.: 1971, Nature 231, 173.

Shukla, P. G. and Wilson, B. G.: 1971, Astrophys. J. 164, 265.

Shulman, S., Fritz, G., Meekins, J. F., Chubb, T. A., Friedman, H., and Henry, R. C.: 1971, Astrophys. J. Letters 166, L9.

Silk, J.: 1970, Space Sci. Rev. 11, 671.

Spitzer, L.: 1956, Astrophys. J. 124, 20.

Strittmatter, P. A., Brecher, K., and Burbidge, G. R.: 1972, Astrophys. J. 174, 91.

Tolbert. C. R.: 1971, Astron. Astrophys. (Suppl.) 3, No. 5.

Toor, A., Price, R., and Seward, F.: 1972, Astrophys. J. Letters 172, L73.

Ulmer, M., Grace, V., Hudson, H. S., and Schwartz, D. A.: 1972, Astrophys. J. 173, 205.

Wolfe, A. M. and Burbidge, G. R.: 1970, Nature 228, 1170.

Woltjer, L.: 1969, Problems of the Crab Nebula, Columbia Astrophys. Lab. Contra. 280-4501, No. 15. Woolf, N.: 1967, Astrophys. J. 148, 287.

Yentis, D. J., Novick, R., and Vanden Bout, P.: 1972, Astrophys. J. 177 (in press). 